\title{
Synthesis of 1-Naphthol by a Natural Peroxygenase Engineered by Directed Evolution
}

\author{
Patricia Molina-Espeja, ${ }^{[\mathrm{a}]}$ Marina Cañellas, ${ }^{[\mathrm{b}]}$ Francisco J. Plou, ${ }^{[\mathrm{a}]}$ Martin Hofrichter, ${ }^{[\mathrm{c}]}$ Fatima Lucas $^{[\mathrm{b}]}$ \\ Victor Guallar $^{[\mathrm{b}]}$ and Miguel Alcalde ${ }^{\star[a]}$
}

\begin{abstract}
There is an increasing interest in enzymes that catalyze the hydroxylation of naphthalene under mild conditions and with minimal requirements. To address this challenge, an extracellular fungal aromatic peroxygenase with mono(per)oxygenase activity was engineered to selectively convert naphthalene into 1-naphthol. Mutant libraries constructed by random mutagenesis and DNA recombination were screened for peroxygenase activity on naphthalene while quenching the undesired peroxidative activity on 1-naphthol (one-electron oxidation). The resulting double mutant (G241D-R257K) of this process was characterized biochemically and computationally. The conformational changes produced by directed evolution improved the substrate's catalytic position. Powered exclusively by catalytic concentrations of $\mathrm{H}_{2} \mathrm{O}_{2}$, this soluble and stable biocatalyst has total turnover numbers of 50,000 , with high regioselectivity (97\%) and reduced peroxidative activity.
\end{abstract}

\section{Introduction}

Currently, around 40,000 tons of 1-naphthol are generated each year to fulfill the need to produce herbicides, insecticides, pharmaceuticals and dye precursors. ${ }^{[1,2]}$ The manufacturing process involves the use of hazardous chemical catalysts with low turnover numbers and poor regioselectivity, and it is associated with elevated energy consumption, high costs and the release of harmful waste products. ${ }^{[3-7]}$ Oxygenases that perform regioselective oxyfunctionalization of aromatic rings would offer a greener alternative to production by standard chemical methods. Accordingly, the manufacturing of 1-naphthol by enzymes should ideally take place in one-pot under mild conditions (room temperature, atmospheric pressure and nearly aqueous solution with small amounts of organic co-solvents), while also reducing the energy required and the noxious waste products resulting from chemical synthesis. ${ }^{[8-11]}$ To date, most studies into the biocatalytic synthesis of 1-naphthol have focused on monooxygenases. In particular, over the years P450 monooxygenases have been designed for different purposes, from the selective hydroxylation of alkanes (including terminal

\footnotetext{
[a] Dr P. Molina Espeja, Dr F.J. Plou and Dr M. Alcalde, Department of Biocatalysis, Institute of Catalysis, CSIC Marie Curie 2 L10, 28049 Madrid (Spain). E-mail: malcalde@icp.csic.es.

[b] M. Cañellas, Dr F. Lucas and Dr V. Guallar Joint BSC-CRG-IRB Research Program in Computational Biology, Barcelona Supercomputing Center, Jordi Girona 29, 08034 Barcelona (Spain).

[c] DrM. Hofrichter,

TU Dresden, International Institute Zittau, Department of Bio- and Environmental Sciences, Markt 23, 02763 Zittau (Germany).
}

hydroxylation) to the non-natural olefin cyclopropanation by carbene transfer. Indeed, these enzymes can transform naphthalene into 1-naphthol by either harnessing the peroxide shunt pathway or through their natural $N A D(P) H$ dependent activity. ${ }^{[12-15]}$ More recently, directed evolution of toluene orthomonooxygenase (TOM) with a whole-cell biocatalytic system was described. ${ }^{[16-18]}$ However, the poor enzyme stability and the reliance on expensive redox cofactors and reductase domains have precluded the practical application of these enzymes in specific industrial settings.

Over a decade ago, the first "true natural" aromatic peroxygenase was discovered (EC 1.11.2.1; also referred to as unspecific peroxygenase, UPO). ${ }^{[19]}$ This enzyme was recently classified in the new heme-thiolate peroxidase superfamily (HTP), along with chloroperoxidase (CPO) from Caldariomyces fumago, even though the latter is not capable of transferring oxygen to aromatic rings or $n$-alkanes. ${ }^{[20]}$ Indeed, the properties of UPO resemble those of P450s in terms of the selective $\mathrm{C}-\mathrm{H}$ oxyfunctionalization of organic compounds. However, UPO is an extracellular, highly active and stable enzyme, and it does not depend on expensive redox cofactors or auxiliary flavoproteins. UPO is "fueled" by stoichiometric concentrations of $\mathrm{H}_{2} \mathrm{O}_{2}$, which serves as an enzyme co-oxidant (i.e. primary electron acceptor) as well as a source of oxygen. Thus, with minimal requirements UPO is capable of performing diverse transformations of great complexity and relevance for organic synthesis, such as the hydroxylation of aromatic and aliphatic compounds, the epoxidation of olefins, the $\mathrm{N}$ - and $\mathrm{S}$-oxidation of heterocycles or the cleavage of ethers, to name but a few. ${ }^{[21]}$ Over 300 substrates have already been reported for UPO (a number that is still growing), of which naphthalene can be converted to 1 naphthol through an epoxide intermediate (as previously described for P450s). Apart from the natural mono(per)oxygenase activity via its two-electron monooxygenation similar to that in the $\mathrm{P} 450$ shunt pathway (confirmed by experiments with ${ }^{18} \mathrm{O}$-labeled $\mathrm{H}_{2} \mathrm{O}_{2}$ ), UPO has peroxidative activity towards phenolic substrates and other compounds through an one-electron oxidation route. ${ }^{[22]}$ This convergence of two such activities in the same protein may become a problem when considering industrial applications, since UPO hydroxylation products are always found along with varying amounts of oxidation by-products. This is especially true for aromatic hydroxylation reactions where the product/s (phenolics) released by peroxygenase activity can serve as reducing substrates of the peroxidative activity, the latter promoting the formation of phenoxyl radicals and quinones that can undergo undesired non-enzymatic polymerization and affect the overall production yields.

In this work, we have employed directed evolution to tailor a UPO from the edible mushroom Agrocybe aegerita (AaeUPO1) 
for the efficient regioselective synthesis of 1-naphthol. Mutant libraries were constructed and expressed in yeast, yielding a final variant that was characterized comprehensively, and its use in the reaction to synthesize 1-naphthol was studied in depth. To obtain a detailed atomic explanation of the effects of the mutations, we also performed ligand migration and quantum chemical simulations.

\section{Results and Discussion}

\section{Directed evolution approach}

Our departure point was a UPO mutant (PaDa-I) that we previously created by directed evolution in Saccharomyces cerevisiae. ${ }^{[23]}$ PaDa-I harbors 9 mutations (F12Y-A14V-R15GA21D-V57A-L67F-V75I-I248V-F311L: the mutations in the signal peptide are underlined) that enhance its functional expression in yeast $(8 \mathrm{mg} / \mathrm{L}$ in $S$. cerevisiae and over $200 \mathrm{mg} / \mathrm{L}$ in Pichia pastoris). ${ }^{[24]}$ At the same time this mutant retains strong activity and stability, particularly in terms of temperature and the presence of co-solvents. Here, mutant libraries of UPO were constructed by random mutagenesis, StEP recombination and in vivo shuffling and they were explored for 1-naphthol synthesis. A sensitive ad hoc dual assay was designed to cap the peroxidative activity on 1-naphthol while protecting the peroxygenase activity on naphthalene (Figure 1).

A
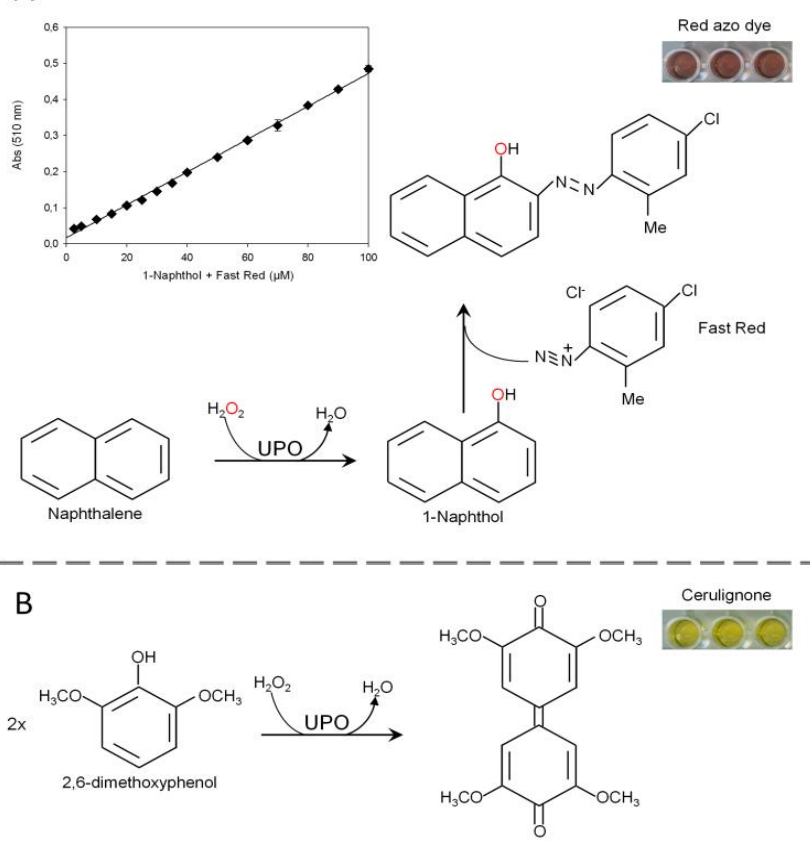

Figure 1. Dual screening assay for naphthalene transformation by UPO. A) For positive selection (peroxygenase activity), mutant libraries were screened with naphthalene such that the formation of the colorless 1-naphthol could be detected using the Fast Red reagent. ${ }^{[25]}$ This compound couples specifically to 1 -naphthol forming a red azo dye that can be readily measured at $510 \mathrm{~nm}$ ( $\varepsilon_{510}$ $=4,700 \mathrm{M}^{-1} \mathrm{~cm}^{-1}$ ), where interference with the culture broth is minimal (the inset shows the linearity and the limit of detection of the assay). B) For the negative criterion of the assay (peroxidative activity), we used a surrogate substrate (the aromatic 2,6-dimethoxyphenol; DMP) that can be oxidized by UPO to form the orange colored cerulignone $\left(\varepsilon_{469}=27,500 \mathrm{M}^{-1} \mathrm{~cm}^{-1}\right)$. The coefficient of variance for both assays was below $12 \%$.

After only two rounds of directed evolution ( 4,000 clones screened), we identified the double mutant G241D-R257K (termed JaWa) that displayed a 2-fold improvement in peroxygenase activity and half the peroxidative activity of the parental type (Supplementary Figure S1).

\section{Biochemical characterization}

PaDa-I and JaWa variants were produced, purified to homogeneity (Reinheitszahl value $[R z]\left[\mathrm{A}_{418} / \mathrm{A}_{280}\right] \sim 2$ ) and characterized biochemically. The general spectral characteristics, molecular weight, $\mathrm{N}$-terminal processing and glycosylation degree were maintained, both for the parental and the mutant enzymes (Table 1).

Table 1. Biochemical features of PaDa-I and JaWa variants.

${ }^{[\mathrm{a}]}$ Estimated by SDS-PAGE; ${ }^{[\mathrm{b}]}$ estimated by MALDI-TOF mass spectrometry;

\begin{tabular}{|c|c|c|}
\hline $\begin{array}{l}\text { Biochemical and spectroscopy } \\
\text { features }\end{array}$ & PaDa-I & JaWa \\
\hline $\mathrm{MW}(\mathrm{Da})^{[\mathrm{a}]}$ & 52,000 & 52,000 \\
\hline $\mathrm{MW}(\mathrm{Da})^{[\mathrm{b}]}$ & 51,100 & 51,100 \\
\hline $\mathrm{MW}(\mathrm{Da})^{[\mathrm{c}]}$ & 35,914 & 35,944 \\
\hline Glycosylation degree (\%) & 30 & 30 \\
\hline $\mathrm{N}$-terminal end & EPGLPPPGPL & EPGLPPPGPL \\
\hline Thermal stability, $T_{50}\left({ }^{\circ} \mathrm{C}\right)^{[\mathrm{d}]}$ & 57.6 & 59.7 \\
\hline $\mathrm{pl}$ & 5.5 & 5.3 \\
\hline Optimum pH for ABTS & 4.0 & 4.0 \\
\hline Optimum $\mathrm{pH}$ for DMP & 6.0 & 6.0 \\
\hline Optimum pH for Naphthalene & 6.0 & 6.0 \\
\hline$R_{\mathrm{Z}},\left(\mathrm{A}_{418} / \mathrm{A}_{280}\right)$ & 1.8 & 2.3 \\
\hline Soret region $(\mathrm{nm})$ & 418 & 418 \\
\hline CT1 (nm) & 570 & 570 \\
\hline CT2 (nm) & 537 & 537 \\
\hline
\end{tabular}

JaWa showed improved thermostability (with a $2^{\circ} \mathrm{C}$ increase in the $T_{50}$-defined as the temperature at which the enzyme retains $50 \%$ of its activity after a 10 min incubation-), and strong stability in the presence of acetonitrile that is used to keep naphthalene in solution (solubility in pure water $31.7 \mathrm{mg} / \mathrm{L}$, (Supplementary Figure S2)). Naphthalene transformation by JaWa and PaDa-I was analyzed by HPLC-PDA. Naphthalene oxygenation by AaeUPO1 occurs through an unstable naphthalene 1,2-oxide intermediate, which undergoes rapid hydrolysis at acid $\mathrm{pH}$ into naphthol (1- and 2-naphthol). ${ }^{[22]}$ Accordingly, we first measured the product distribution after stopping a 15 min reaction with $\mathrm{HCl}$. Both PaDa-I and JaWa showed similar regioselectivity (92\% 1naphthol, $8 \%$ 2-naphthol), while JaWa produced significantly more 1-naphthol (156\% compared to PaDa-I) without detecting the formation of the 1-naphthol oxidation product, 1,4naphthoquinone (Figure 2A). A similar behavior was observed when we followed the reaction for longer ( $270 \mathrm{~min}$ at $\mathrm{pH} 7.0$

Figure 2. Naphthalene conversion by evolved UPOs. A) Product distribution after a 15 min reaction stopped with $\mathrm{HCl}$ (PaDa-I, black bars; JaWa, grey bars). Reactions were performed at room temperature and the reaction mix contained $6.6 \mathrm{nM}$ of purified enzyme, $1 \mathrm{mM}$ naphthalene, $20 \%$ acetonitrile and $1 \mathrm{mM} \mathrm{H}_{2} \mathrm{O}_{2}$ in $100 \mathrm{mM}$ potassium phosphate buffer $\mathrm{pH} 7.0$ (1 mL final volume). 


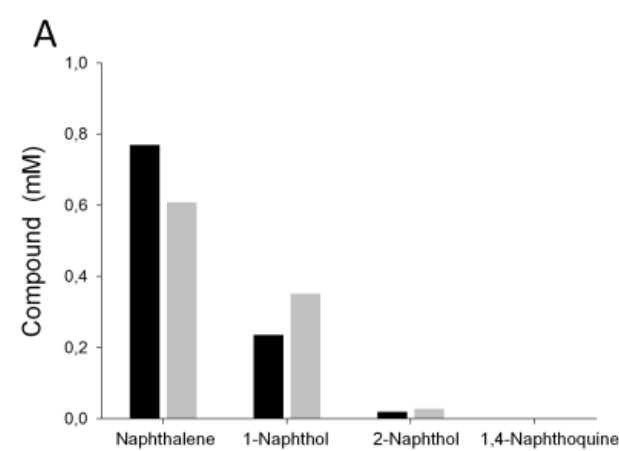

C

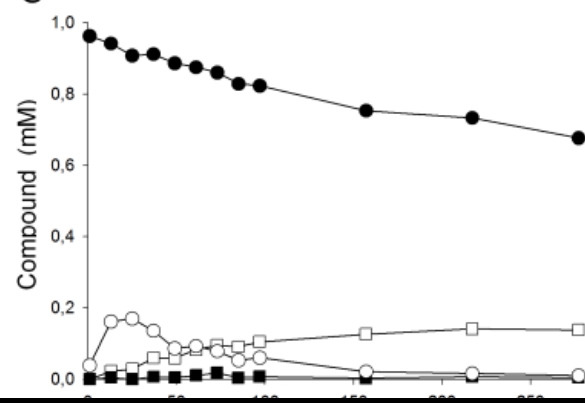

\begin{tabular}{|c|c|c|}
\hline Substrate & Kinetieneofminhts & PaDa-I \\
\hline ABTS & $\begin{array}{c}K_{\mathrm{m}}(\mu \mathrm{M}) \\
K_{\text {cat }}\left(\mathrm{s}^{-1}\right) \\
k_{\mathrm{cat}} / K_{\mathrm{m}}\left(\mathrm{s}^{-1} \mathrm{M}^{-1}\right)\end{array}$ & $\begin{array}{c}48.0 \pm 4.5 \\
395 \pm 13 \\
8.2 \times 10^{6} \pm 6 \times 10^{5}\end{array}$ \\
\hline DMP & $\begin{array}{c}K_{\mathrm{m}}(\mu \mathrm{M}) \\
K_{\text {cat }}\left(\mathrm{s}^{-1}\right) \\
k_{\mathrm{cat}} / K_{\mathrm{m}}\left(\mathrm{s}^{-1} \mathrm{M}^{-1}\right)\end{array}$ & $\begin{array}{c}126 \pm 14 \\
68 \pm 2 \\
5.4 \times 10^{5} \pm 4.8 \times 10^{4}\end{array}$ \\
\hline Naphthalene & $\begin{array}{c}K_{\mathrm{m}}(\mu \mathrm{M}) \\
k_{\text {cat }}\left(\mathrm{s}^{-1}\right) \\
k_{\text {cat }} / K_{\mathrm{m}}\left(\mathrm{s}^{-1} \mathrm{M}^{-1}\right)\end{array}$ & $\begin{array}{c}578 \pm 106 \\
229 \pm 17 \\
4.0 \times 10^{5} \pm 4.0 \times 10^{6}\end{array}$ \\
\hline $\mathrm{H}_{2} \mathrm{O}_{2}$ & $\begin{array}{c}K_{\mathrm{m}}(\mu \mathrm{M}) \\
K_{\text {cat }}\left(\mathrm{s}^{-1}\right) \\
k_{\text {cat }} / K_{\mathrm{m}}\left(\mathrm{s}^{-1} \mathrm{M}^{-1}\right)\end{array}$ & $\begin{array}{c}486 \pm 55 \\
238 \pm 8 \\
5.0 \times 10^{5} \pm 4.2 \times 10^{4}\end{array}$ \\
\hline
\end{tabular}

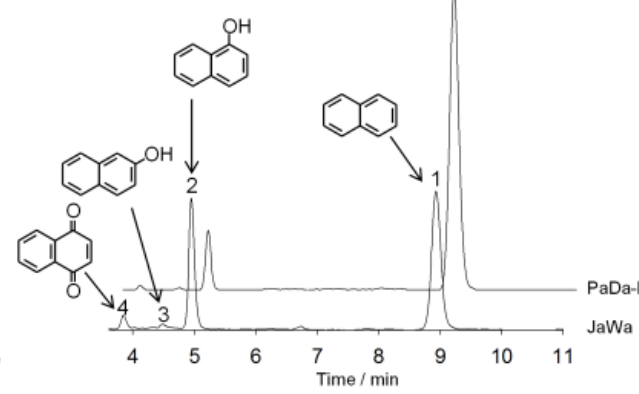

D

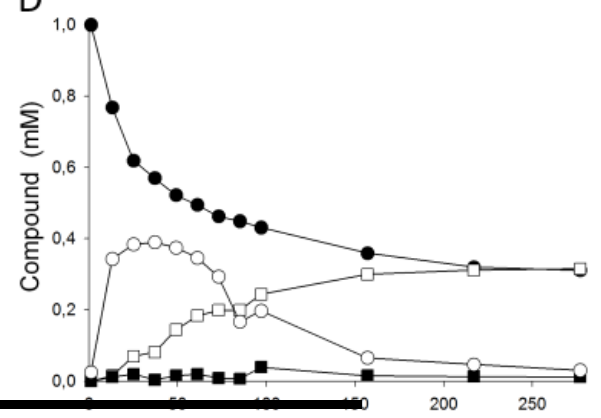

similar when analyzed by mass spectrometry (Supplementary Figure S3). Indeed, we calculated total turnover numbers (TTN) of approximately 50,000 for JaWa as opposed to 20,000 for the PaDa-I parental type. When the kinetic parameters of the peroxygenative and peroxidative activities of both enzymes were determined (Table $1)$, the $k_{\text {cat }} / K_{\mathrm{m}}$ (catalytic efficiency) of JaWa for naphthalene hydroxylation increased 1.5-fold, while the peroxidative activity was partially suppressed, with a dramatic 3- to 12-fold decrease in catalytic efficiencies for the peroxidative substrates 2,6-DMP and ABTS (2,2'-azino-bis(3ethylbenzothiazoline-6-sulfonic acid), respectively. Moreover, the $k_{\text {cat }} / K_{\mathrm{m}}$ of $\mathrm{H}_{2} \mathrm{O}_{2}$ with benzyl alcohol as reducing substrate was also affected.$$
181 \pm 22
$$

$$
\begin{aligned}
125 & \pm 5 \\
6.9 \times 10^{5} & \pm 6.3 \times 10^{4} \\
866 & \pm 108 \\
142 & \pm 8 \\
1.6 \times 10^{5} & \pm 1.2 \times 10^{4} \\
127 & \pm 27 \\
78 & \pm 3 \\
6.2 \times 10^{5} & \pm 1.1 \times 10^{5} \\
1,250 & \pm 300 \\
447 & \pm 40 \\
3.6 \times 10^{5} & \pm 5.9 \times 10^{4}
\end{aligned}
$$

To ascertain how the decrease in the peroxidative activity affected the TTN for the hydroxylation of naphthalene, we measured the turnover rate $((\mu \mathrm{mol}$ product) ( $\mu \mathrm{mol}$ enzyme $)^{-1} \mathrm{~min}^{-1}$ ) for the conversion of 1-naphthol to 1,4-naphthoquinone by HPLC. Although the catalytic rate of $\mathrm{PaDa}-\mathrm{I}$ for 1-naphthol was already low $\left(200 \mathrm{~min}^{-1}\right)$, it dropped further to 92 $\mathrm{min}^{-1}$ in JaWa, with a $\sim 1.5$-fold reduction in the molar ratio 1,4-naphthoquinone:1-naphthol (Figure 3A).

After $15 \mathrm{~min}$, the reaction was stopped with $20 \mu \mathrm{L}$ of $37 \% \mathrm{HCl}$ and analyzed by HPLC-PDA. B) HPLC elution profiles after a reaction time of $270 \mathrm{~min}(1$, naphthalene; 2, 1-naphthol; 3, 2-naphthol; 4, 1,4-naphthoquinone). C) and D) Time courses of the reactions over $270 \mathrm{~min}$ at pH 7.0 with PaDa-I and JaWa, respectively (without stopping the reaction by adding $\mathrm{HCl}$ ). Black circles, naphthalene; white circles, naphthalene 1,2-oxide; white squares, 1-naphthol; black squares, 2-naphthol. The total turnover numbers (TTN, reported as $\mu \mathrm{mol}$ product/ $/ \mathrm{mol}$ enzyme) were estimated from the 1 -naphthol concentration after $270 \mathrm{~min}$.

Table 2. Kinetic constants for the PaDa-l and JaWa variants. The kinetic constants for ABTS were measured in $100 \mathrm{mM}$ sodium phosphate/citrate buffer $\mathrm{pH} 4.0$ containing $2 \mathrm{mM} \mathrm{H}_{2} \mathrm{O}_{2}$, while the rest of the substrates were tested in $100 \mathrm{mM}$ potassium phosphate $\mathrm{pH} 7.0$ containing 2 $\mathrm{mM} \mathrm{H}_{2} \mathrm{O}_{2}$ (DMP) or $1 \mathrm{mM} \mathrm{H}_{2} \mathrm{O}_{2}$. Varying amounts of naphthalene were added from stocks in acetonitrile to give a final co-solvent concentration of $20 \% \mathrm{v} / \mathrm{v}$.

For $\mathrm{H}_{2} \mathrm{O}_{2}$, benzyl alcohol was used as a reducing substrate at the corresponding saturated conditions.

without stopping the reaction by adding $\mathrm{HCl}$ ), indicating that the chemical conversion of naphthalene 1,2-oxide into naphthols also occurs at neutral $\mathrm{pH}$, although at lower rates. Moreover, traces of 1,4-naphthoquinone were detected (Figure 2B, C, D). While the formation of the epoxide intermediate by both enzymes reached a plateau at $\sim 40 \mathrm{~min}$ (due to oxidative damage caused by $\mathrm{H}_{2} \mathrm{O}_{2}$ ), the regioselectivity was enhanced to $97 \%$ of 1 -naphthol. This result is in good agreement with the loss of selectivity observed when $\mathrm{HCl}$ is used to stop the reaction due to the increased reactivity of the epoxide intermediate in an acid environment. ${ }^{[22]}$ In terms of production yields, the differences between the mutants were even more pronounced (i.e. 0.14 and $0.32 \mathrm{mM}$ of 1 -naphthol, for PaDa-I and JaWa, respectively) while the products produced were
This effect could also be visualized as the polymeric products formed through the non-enzymatic coupling of the phenoxyl radicals produced by $\mathrm{PaDa}-\mathrm{I}$ are colored (inset in Figure $3 \mathrm{~A}$ ). ${ }^{[12]}$ It has been reported that UPO may resemble CPO in terms of the presence of different oxidation sites for peroxidative activity. ${ }^{[26]}$ Thus, we examined the recent AaeUPO1 crystal structure to find a means to suppress alternative peroxidative pathways. ${ }^{[27]} \mathrm{QM} / \mathrm{MM}$ calculations identified Trp24 as the highest oxidizable surface residue (see computational analysis below); hence, we constructed site-directed variants using the PaDa-I and JaWa variants as templates. The corresponding PaDa-IW24F and JaWa-W24F mutants were compared: Irrespective of the variant and the reducing substrate tested, the W24F mutation decreased the peroxidative activity by $\sim 60 \%$ (Figure $3 \mathrm{~B})$. However, this mutation also affected the peroxygenase activity, with a $\sim 50 \%$ drop in the hydroxylation of naphthalene and the fission of 5-nitro-1,3-benzodioxole (NBD), suggesting that Trp24 may also play a role in the peroxygenase activity of UPO.

Figure 3. A) Turnover rates for 1-naphthol oxidation. Reactions ( $1 \mathrm{~mL}$ final volume) were carried out at room temperature and contained $1 \mathrm{mM}$ naphthol, $1 \mathrm{mM} \mathrm{H}_{2} \mathrm{O}_{2}, 20 \%$ acetonitrile and purified enzyme ( $40 \mathrm{nM}$ ) in $100 \mathrm{mM}$ potassium phosphate $\mathrm{pH}$ 7.0. 1-N, 1-naphthol; 1,4-NQ, 1-4-naphthoquinone. The reactions were performed in triplicate and were stopped by adding $\mathrm{HCl}$ $(\mathrm{pH}<1)$ after different times $(60$ to $600 \mathrm{~s})$. Inset: Colored polymeric products from 1,4-naphthoquinone; 1, PaDa-l; 2, JaWa. B) Activities of W24F sitedirected variants. The buffer used was $100 \mathrm{mM}$ potassium phosphate $\mathrm{pH} 7.0$, except for ABTS where the buffer was $100 \mathrm{mM}$ sodium phosphate/citrate $\mathrm{pH}$ 4.0. The substrate concentrations were: $0.5 \mathrm{mM}$ naphthalene, $1 \mathrm{mM} \mathrm{NBD}$, $3 \mathrm{mM} \mathrm{DMP}$ and $0.3 \mathrm{mM}$ ABTS. In all cases, $1 \mathrm{mM} \mathrm{H}_{2} \mathrm{O}_{2}$ and $15 \%$ acetonitrile 


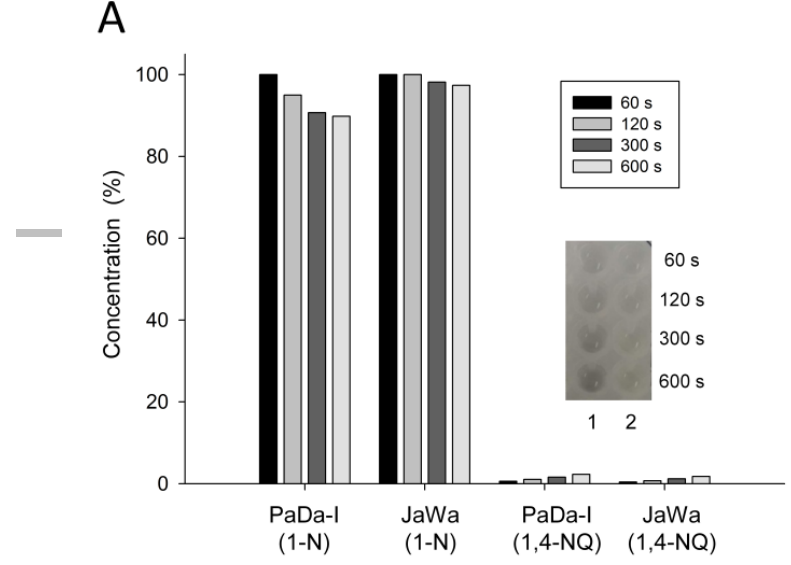

B

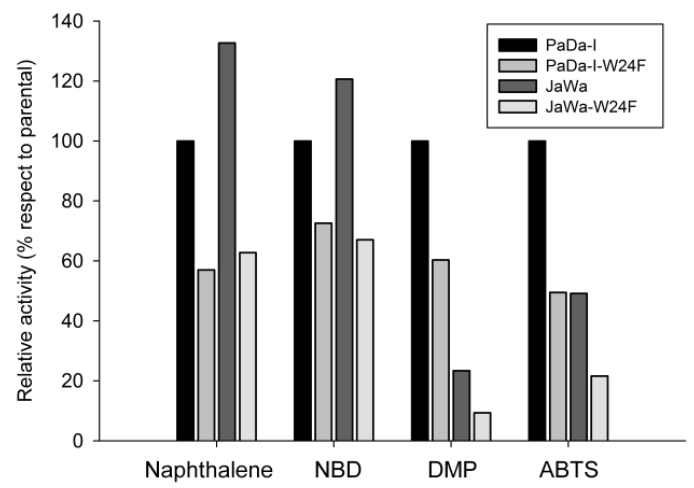

were added to complete the reaction mixture. For naphthalene activity, a Fast Red assay was used (after 10 min of reaction, Fast Red was added at a final concentration of $0.5 \mathrm{mM}$, and when a red color appeared and was stabilized, the absorbance was measured). The molar extinction coefficients are: Naphthalene+Fast Red, $\varepsilon_{510}=4,700 \mathrm{M}^{-1} \mathrm{~cm}^{-1}$; NBD, $\varepsilon_{425}=9,700 \mathrm{M}^{-1} \mathrm{~cm}^{-1}$; DMP, $\varepsilon_{469}=27,500 \mathrm{M}^{-1} \mathrm{~cm}^{-1}$ and ABTS, $\varepsilon_{418}=36,000 \mathrm{M}^{-1} \mathrm{~cm}^{-1}$.

\section{Computational analysis}

The JaWa mutations were mapped in the AaeUPO1 structure with a characteristic binding pocket where the hydrophobic Phe69-Phe121-Phe199 triad is involved in the correct orientation of aromatic compounds (Figure 4, Supplementary Figure S4). The G241D mutation is located at the entrance of the heme access channel, while R257K is situated at the surface of the protein, far from any relevant catalytic sites. In order to understand the atomic mechanistic details responsible for the observed differences in catalytic activity between $\mathrm{PaDa}-\mathrm{I}$ and JaWa variants we turned to molecular modeling PELE (protein energy landscape exploration) software ${ }^{[28]}$ was used to

A
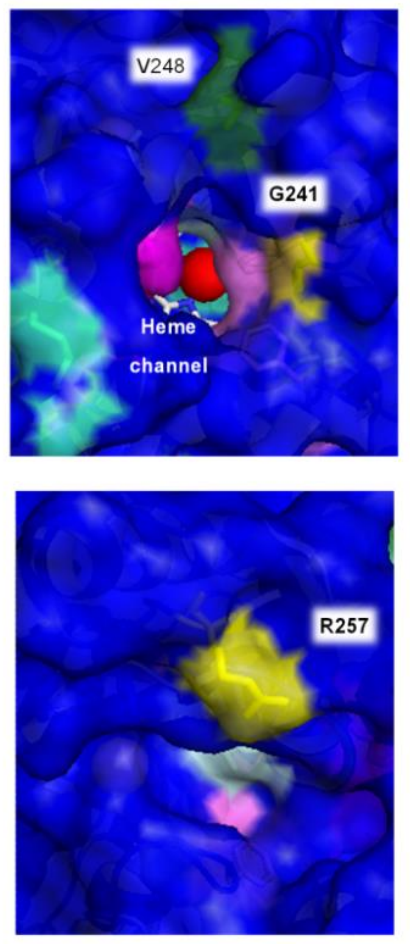

B
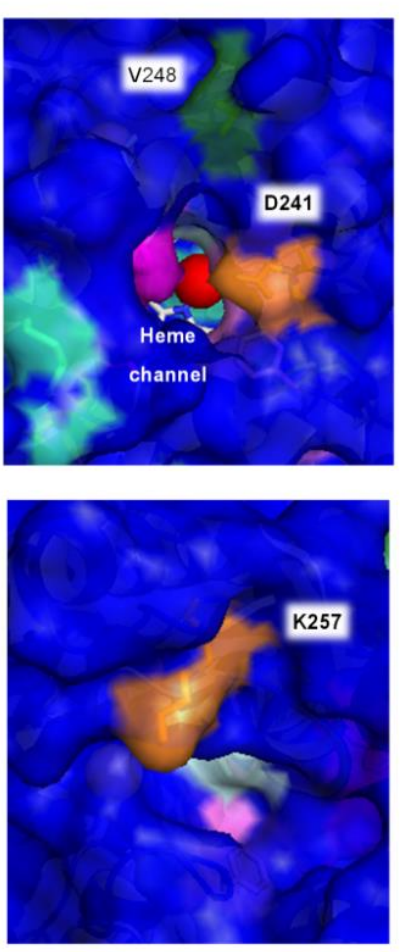

investigate naphthalene and DMP's interaction energy profiles, both at the heme binding site and on the protein's surface.

Peroxidative activity: Global ligand migration exploration, aimed at identifying the most favorable binding sites, found the heme entrance, Trp24 and Tyr29 as the most favorable minima. Then, following the protocol employed previously to identify catalytically active surface residues in a dye-decolorizing peroxidase ${ }^{[29]}, \mathrm{QM} / \mathrm{MM}$ calculations were performed on wild-type AaeUPO1. All potential surface oxidation sites ( 9 tyrosines and 1 tryptophan) were included in the quantum region, where one electron was removed and spin density computed. Furthermore, pairwise comparison was performed with the residues that showed a clear preference to be oxidized, Trp24 and Tyr47. Results showed Trp24 to be the most favorable site for peroxidative activity as described above (see Supplementary Figure S5A).

Figure 4. Mutations in the evolved UPO. Protein modeling was based on the AaeUPO1 crystal structure (PDB accession number 2YOR). A) PaDa-l; B) JaWa. The G241D and R257K substitutions are depicted in yellow and orange. The V248 mutation (dark green) comes from previous directed evolution campaigns. ${ }^{[23]}$ The Phe residues involved in accommodating substrates at the catalytic pocket are highlighted in pink, the Cys residues are in light green (C36 axial ligand and the C278-C319 disulfide bridge), the R189 component of the acid-base pair involved in the catalysis is tagged in light blue and the $\mathrm{Fe}^{3+}$ of heme is represented as a red sphere.

Peroxygenase activity: To rationalize the effect of the two introduced mutations on $k_{\mathrm{cat}}$ and $K_{\mathrm{m}}$, ligand exploration at the
A

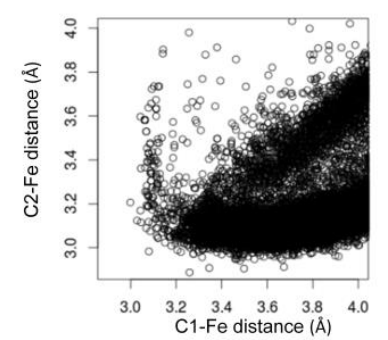

B

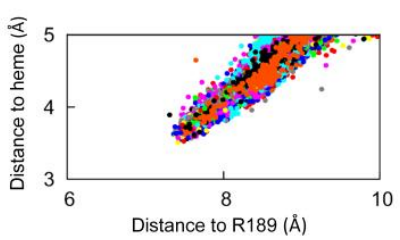

JaWa
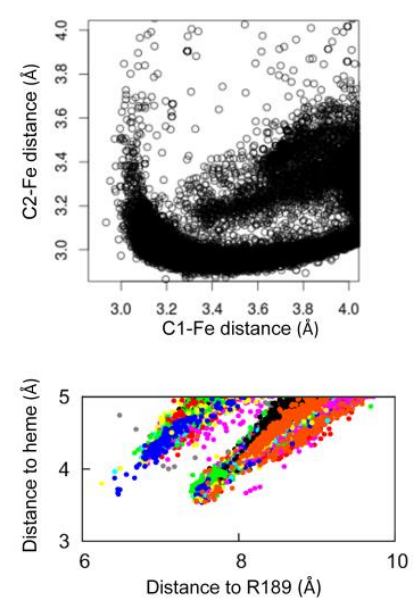

heme site was performed with PELE using the two AaeUPO1 variants: JaWa and PaDa-I. Additionally, $\mathrm{QM} / \mathrm{MM}$ calculations were carried out to investigate the mutations' effect on naphthalene oxygenation.

i) Structural variations. Heme binding site PELE simulations with naphthalene show that in JaWa variant, G241D mutation induces a $\sim 20^{\circ}$ rotation in the aspartate backbone dihedral (Supplementary Figure S5B) leading to a shift on the loop where it is located. Along with this rotation we observed the disruption of the hydrogen bond between Thr198 and Gly241 (highly stable in PaDa-I, 86\% residence time versus $0 \%$ in JaWa). These changes create space for the concomitant displacement of the $\alpha$-helix that hosts the catalytic acid-base (Arg189-Glu196) pair (Figure 5). 


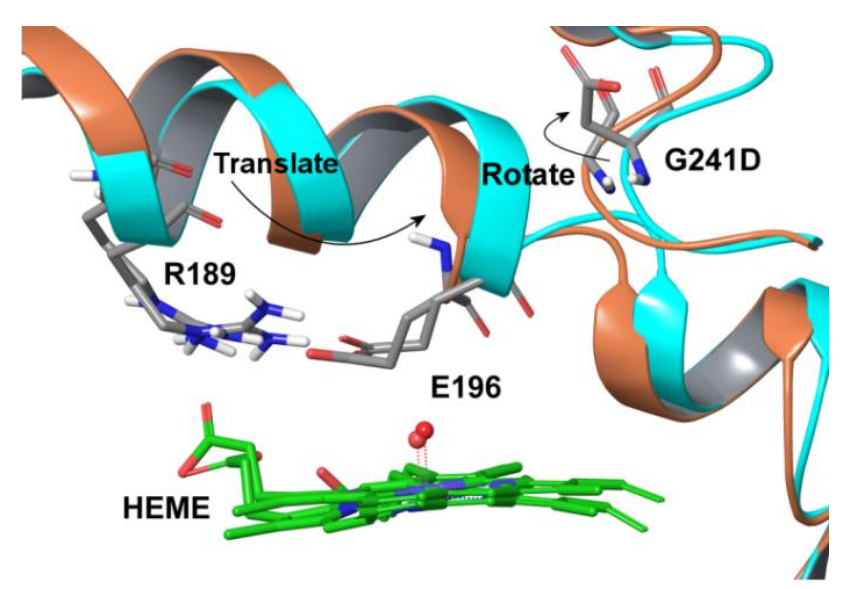

Figure 5. G124D induced conformational changes. Variation of the 241residue dihedral along PELE simulations. CB-CA-C-O dihedral was measured for JaWa (cyan) and HA3-CA-C-O for PaDa-I (orange). The average value for JaWa is $-60.2^{\circ}$ and $-43.6^{\circ}$ for PaDa-I.

ii) Naphthalene binding. Previously mentioned structural changes (induced by G241D), along with other minor variations in the protein's structure, caused a redistribution of the binding site occupation in JaWa mutant. Ideally, in order to facilitate epoxide intermediate formation, distances between $\mathrm{C} 1$ and $\mathrm{C} 2$ naphthalene carbons and the heme catalytic oxygen should be below $3 \AA$ for optimum reaction. As seen in Figure $6 A$, analysis of naphthalene's binding site position along PELE simulations show an increased population of optimum catalytic orientations in JaWa (lower naphthalene-heme distances). This is in agreement with the 4.5-times decreased $K_{\mathrm{m}}$ for this variant (Table 2).

iii) Hydroxylation of naphthalene. Mutation-induced structural variations not only have a repercussion on naphthalene binding site orientation, but also on its hydroxylation. The $\alpha$-helix structural changes induce a new Arg189 alternate position, closer to the substrate (Figure 6B). To compute the effect of this electrostatic environment change on naphthalene's oxidation we turned again to $\mathrm{QM} / \mathrm{MM}$ calculations. Naphthalene ionization energies were computed for both $\mathrm{PaDa}-\mathrm{I}$ and JaWa variants. Results show that the increase in the substrate's positive electrostatic environment increases naphthalene's ionization energy (from $213.55 \mathrm{kcal} / \mathrm{mol}$ in PaDa-I to $214.57 \mathrm{kcal} / \mathrm{mol}$ in JaWa), leading to a higher activation barrier, as shown in previous studies ${ }^{[30,31]}$ and in agreement with JaWa lower $k_{\text {cat }}$ observed experimentally.

Figure 6. A) Distances between $\mathrm{C} 1 / \mathrm{C} 2$ naphthalene carbons (hydroxylated carbons) and the heme catalytic oxygen showing a larger substrate population for optimum reactive distances $(<3 \AA$ ) in JaWa. B) Variation of naphthalene's center of mass (COM) to heme oxygen (compound I) vs. naphthalene's COM to $\mathrm{CZ}$ atom in R189 in PaDa-I and JaWa along PELE simulations. Results show that in the case of JaWa the arginine side chain is in average closer to the substrate.

On the other hand, The R257K mutation was not expected to affect the hydroxylation of naphthalene but further PELE simulations with DMP show an additional access to the active heme site, close to this residue (not present in naphthalene), which could affect both the $K_{\mathrm{m}}$ and $k_{\text {cat }}$. Moreover, it is known that some peroxidases have different inlets at the surface of the protein that serve for the one-electron oxidation of reducing substrates through a long-range electron transfer pathway towards the heme domain, as described here for W24F variants. ${ }^{[32]}$ The R257K substitution may affect some of these enzyme circuits, possibly offering benefits in terms of improved thermostability through local rearrangements in secondary structures. Indeed, both the G241D and R257K mutations modified the local B factor profiles (Supplementary Figure S6).

\section{Conclusions}

To date, over 1,000 UPO-like genes from fungi have been mined from genomic databases, highlighting their widespread distribution in the fungal kingdom. Nevertheless, only three wildtype UPOs have been biochemically characterized and published (from Coprinellus radians, Marasmius rotula and from the abovementioned $A$. aegerita). Some other UPOs have been identified (e.g., from Chaetomium sp.) but they have not yet been characterized in detail. ${ }^{[3]}$ More recently, UPO genes from the genomes of Coprinopsis cinerea and from a soil mold were expressed heterologously. ${ }^{[21]}$ The AaeUPO1 engineered here shows the highest selectivity and TTN for the production of 1 naphthol hitherto reported in this enzyme superfamily. Readily secreted in an active, soluble and very stable form, this heterologous enzyme performs selective aromatic oxygenations in the absence of $\mathrm{NAD}(\mathrm{P}) \mathrm{H}$ cofactors and reductase domains. Its self-sufficient mono(per)oxygenase activity, along with a diminished peroxidative activity, make this UPO mutant a valuable biocatalyst for future synthetic applications.

\section{Experimental Section}

Reagents and enzymes: ABTS (2,2'-azino-bis(3-ethylbenzothiazoline-6sulfonic acid)), DMP (2,6-dimethoxyphenol), benzyl alcohol, 1-naphthol, 2-naphthol, 1,4-naphthoquinone, Fast Red TR Salt hemi(zinc chloride) salt, Taq DNA polymerase and the Yeast Transformation kit were purchased from Sigma-Aldrich (Saint Louis, MO, USA). NBD (5-nitro-1,3benzodioxole) was acquired from TCI America (Portland, OR, USA) and naphthalene from Acros Organics (Geel, Belgium). The Genemorph II Random Mutagenesis kit (Mutazyme II) was obtained from Agilent Technologies (Santa Clara, CA, USA) and the high fidelity DNA polymerase iProof was acquired from Bio-Rad (Hercules, CA, USA). The $\mathrm{BamHI}$ and Xhol restriction enzymes were purchased from New England Biolabs (Ipswich, MA, USA) and the protease deficient $S$. cerevisiae strain BJ5465 was from LGCPromochem (Barcelona, Spain). The Zymoprep Yeast Plasmid Miniprep kit and Zymoclean Gel DNA Recovery kit were from Zymo Research (Orange, CA, USA). The NucleoSpin Plasmid kit was purchased from Macherey-Nagel (Düren, Germany) and the oligonucleotides were synthesized by Isogen Life Science (Barcelona, Spain). All chemicals were reagent-grade purity.

Laboratory evolution: The parental PaDa-I was obtained as described elsewhere. ${ }^{[23]}$ After each round of directed evolution, the PCR products were loaded onto a preparative agarose gel and purified using the Zymoclean Gel DNA Recovery kit. The recovered DNA fragments were cloned under the control of the GAL1 promoter of the pJRoC30 expression shuttle vector, using BamHI and Xhol to linearize the plasmid and remove the parental gene. The linearized vector was loaded onto a low melting point preparative agarose gel and purified with the Zymoclean Gel DNA Recovery kit. 
First generation. Using PaDa-I as parental type, error prone PCR was carried out in a reaction mix ( $50 \mu \mathrm{L}$ final volume) containing $3 \%$ dimethyl sulfoxide (DMSO), $0.37 \mu \mathrm{M}$ RMLN (5'-cctctatactttaacgtcaagg-3'), $0.37 \mu \mathrm{M}$ RMLC (5'-gcttacattcacgccctccc-3'), $0.8 \mathrm{mM}$ deoxynucleotide triphosphates (dNTPs, $0.2 \mathrm{mM}$ each), $0.05 \mathrm{U} / \mathrm{\mu L}$ Mutazyme ॥ (Genemorph II kit) and 2,822 ng of the template (plasmid pJRoC30 containing PaDa-l, $300 \mathrm{ng}$ of the target DNA). The mutagenic PCR was performed on a gradient thermocycler (Mycycler, Bio-Rad, USA) using the following cycles: $95^{\circ} \mathrm{C}$ for $2 \min (1 \mathrm{cycle}) ; 94^{\circ} \mathrm{C}$ for $45 \mathrm{~s}, 53^{\circ} \mathrm{C}$ for $45 \mathrm{~s}$ and $74^{\circ} \mathrm{C}$ for $3 \mathrm{~min}$ ( $\left.28 \mathrm{cycles}\right)$; and $74^{\circ} \mathrm{C}$ for $10 \mathrm{~min}$ ( 1 cycle). The PCR products $(200 \mathrm{ng})$ were mixed with the linearized plasmid $(100 \mathrm{ng})$, and transformed into competent $S$. cerevisiae cells for in vivo shuffling and cloning using the Yeast Transformation kit. Transformed cells were plated on synthetic complete (SC) drop-out plates and incubated for 3 days at $30^{\circ} \mathrm{C}$. Grown colonies were selected and subjected to the dual high-throughput screening (HTS) assay and additional re-screenings, as described below.

Second generation. The best mutants obtained from the first generation were used to perform mutagenic StEP ${ }^{[34]}$ combined with in vivo shuffling. The PCR reaction contained: $3 \%$ DMSO, $90 \mathrm{nM}$ RMLN, $90 \mathrm{nM}$ RMLC, $0.3 \mathrm{mM}$ dNTPs (0.075 mM each), $0.05 \mathrm{U} / \mu \mathrm{L}$ Taq DNA polymerase and $16 \mathrm{ng}$ of the template (pJRoC30 containing the four best mutants of the first generation). The PCR was performed on a gradient thermocycler using the following cycles: $95^{\circ} \mathrm{C}$ for $5 \mathrm{~min}(1 \mathrm{cycle}) ; 94^{\circ} \mathrm{C}$ for $30 \mathrm{~s}, 55^{\circ} \mathrm{C}$ for $20 \mathrm{~s}$ (90 cycles). The PCR products were mixed with the linearized vector (200 $\mathrm{ng}$ of each PCR product and $100 \mathrm{ng}$ of linearized plasmid), transformed into competent $S$. cerevisiae cells and treated as for the first generation.

W24F site-directed variants. PCR reactions for each variant (PaDa-I and JaWa) were carried out using the primers: F24FOR, 5'ctcacccattraagccgcttcgacctggcgatattcgtggac-3'; and F24REV, 5'gtccacgaatatcgccaggtcgaagcggcttaaatgggtgag-3' (mutated bases are underlined). The PCR reactions contained: (i) $50 \mu \mathrm{L}$ final volume, $3 \%$ DMSO, $0.5 \mu \mathrm{M}$ RMLN, $0.5 \mu \mathrm{M}$ F24REV, $1 \mathrm{mM}$ dNTPs (0.25 mM each), $0.02 \mathrm{U} / \mathrm{\mu L}$ high fidelity DNA polymerase iProof and $10 \mathrm{ng}$ of the templates; and (ii) $50 \mu \mathrm{L}$ final volume, 3\% DMSO, $0.5 \mu \mathrm{M}$ F24FOR, 0.5 $\mu \mathrm{M}$ RMLC, $1 \mathrm{mM}$ dNTPs (0.25 mM each), $0.02 \mathrm{U} / \mu \mathrm{L}$ high fidelity DNA polymerase iProof and $10 \mathrm{ng}$ of the templates. The thermocycler parameters were: (i) $98^{\circ} \mathrm{C}$ for $30 \mathrm{~s}(1 \mathrm{cycle}), 98^{\circ} \mathrm{C}$ for $10 \mathrm{~s}, 47^{\circ} \mathrm{C}$ for $25 \mathrm{~s}$, $72^{\circ} \mathrm{C}$ for $15 \mathrm{~s}$ ( $\left.28 \mathrm{cycles}\right)$, and $72^{\circ} \mathrm{C}$ for $10 \mathrm{~min}(1 \mathrm{cycle})$; or (ii) $98^{\circ} \mathrm{C}$ for $30 \mathrm{~s}$ ( 1 cycle), $98^{\circ} \mathrm{C}$ for $10 \mathrm{~s}, 58^{\circ} \mathrm{C}$ for $25 \mathrm{~s}, 72^{\circ} \mathrm{C}$ for $45 \mathrm{~s}$ ( 35 cycles), and $72^{\circ} \mathrm{C}$ for $10 \mathrm{~min}$ ( $1 \mathrm{cycle}$ ). PCR products belonging to each template (200 $\mathrm{ng}$ each) were mixed with the linearized vector $(100 \mathrm{ng})$, and transformed into $S$. cerevisiae for in vivo gene reassembly and cloning by IVOE. ${ }^{[35]}$

High-throughput screening assay. Individual clones were picked and inoculated in sterile 96-well plates (Greiner Bio-One GmbH, Germany), referred to as master plates, containing: $200 \mu \mathrm{L}$ of minimal expression medium per well $(100 \mathrm{~mL} 6.7 \%$ filtered yeast nitrogen base, $100 \mathrm{~mL} 19.2$ $\mathrm{g} / \mathrm{L}$ filtered yeast synthetic drop-out medium supplement without uracil, $67 \mathrm{~mL} 1 \mathrm{M}$ filtered potassium phosphate buffer $\mathrm{pH} 6.0,111 \mathrm{~mL} 20 \%$ filtered galactose, $22 \mathrm{~mL}$ filtered $\mathrm{MgSO}_{4} 0.1 \mathrm{M}, 31.6 \mathrm{~mL}$ absolute ethanol, $1 \mathrm{~mL} 25 \mathrm{~g} / \mathrm{L}$ filtered chloramphenicol and $d d \mathrm{H}_{2} \mathrm{O}$ to $1,000 \mathrm{~mL}$ ). In each plate, column number 6 was inoculated with the corresponding parental type and one well ( $\mathrm{H} 1$-control) was inoculated with untransformed $S$. cerevisiae cells. The plates were sealed to prevent evaporation and incubated at $30^{\circ} \mathrm{C}, 220 \mathrm{RPM}$ and $80 \%$ relative humidity in a shaker (Minitron, INFORS, Switzerland) for five days. The master plates were centrifuged (Eppendorf 5810R centrifuge, Germany) for $10 \mathrm{~min}$ at 3,500
RPM and $4^{\circ} \mathrm{C}$. Aliquots of the supernatants $(20 \mu \mathrm{L})$ were transferred from the master plates to two replica plates by using a liquid handler robotic station Freedom EVO (Tecan, Switzerland). The reaction mixture (180 $\mu \mathrm{L})$ with DMP or naphthalene was added to each replica plate with the help of a pipetting robot (Multidrop Combi Reagent Dispenser, Thermo Scientific, USA). The DMP reaction mixture contained $100 \mathrm{mM}$ potassium phosphate buffer at $\mathrm{pH} 7.0,3 \mathrm{mM} \mathrm{DMP}$ and $1 \mathrm{mM} \mathrm{H}_{2} \mathrm{O}_{2}$. At the same time, the same screening was carried out but with the addition of $10 \%$ of acetonitrile to assess possible changes in activity due to resistance to this organic co-solvent, needed for naphthalene solution. The reaction mixture with naphthalene contained $100 \mathrm{mM}$ potassium phosphate buffer $\mathrm{pH} 7.0,0.5 \mathrm{mM}$ naphthalene, $10 \%$ acetonitrile and $1 \mathrm{mM} \mathrm{H}_{2} \mathrm{O}_{2}$. The plates were stirred briefly and the initial absorptions at $469 \mathrm{~nm}$ and 510 $\mathrm{nm}$, for DMP and naphthalene respectively, were recorded in the plate reader (SPECTRAMax Plus 384, Molecular Devices, Sunnyvale, CA). After a reaction time of $10 \mathrm{~min}$, Fast Red (Fast Red TR Salt hemi(zinc chloride) salt, $20 \mu \mathrm{L}$ ) was added to each well (final concentration of 0.5 $\mathrm{mM}$ ) and the plates were incubated at room temperature until a red (naphthalene-Fast Red) or orange (DMP) color developed and the absorption was measured again. The values were normalized against the parental type in the corresponding plate. To rule out false positives, two re-screenings were carried out and a third re-screening was performed in order to assess the kinetic stability (the protocol for the re-screenings and the studies of kinetic stability $-T_{50}$ - are described elsewhere ${ }^{[23]}$ ).

Biochemical characterization: PaDa-I and JaWa variants were produced and purified as described in ${ }^{[23]}$.

Steady-state kinetic constants. ABTS kinetic constants for UPO were estimated in $100 \mathrm{mM}$ sodium phosphate/citrate buffer $\mathrm{pH} 4.0$ containing 2 $\mathrm{mM} \mathrm{H} \mathrm{H}_{2}$, and for the rest of the substrates in $100 \mathrm{mM}$ potassium phosphate buffer $\mathrm{pH} 7.0$ containing $2 \mathrm{mM} \mathrm{H}_{2} \mathrm{O}_{2}$ (DMP) or $1 \mathrm{mM} \mathrm{H}_{2} \mathrm{O}_{2}$ (naphthalene, in $20 \%$ of acetonitrile - final concentration). For $\mathrm{H}_{2} \mathrm{O}_{2}$, benzyl alcohol was used as a reducing substrate at the corresponding saturated conditions. Reactions were performed in triplicate and substrate oxidations were followed through spectrophotometric changes (ABTS, $\varepsilon_{418}=36,000 \mathrm{M}^{-1} \mathrm{~cm}^{-1}$; DMP, $\varepsilon_{469}=27,500 \mathrm{M}^{-1} \mathrm{~cm}^{-1}$; naphthalene $\varepsilon_{303}=2,010 \mathrm{M}^{-1} \mathrm{~cm}^{-1}$; benzyl alcohol; $\varepsilon_{280}=1,400 \mathrm{M}^{-1} \mathrm{~cm}^{-1}$ ). Naphthalene kinetics were performed following the protocol described elsewhere ${ }^{[36]}$. To calculate the $K_{\mathrm{m}}$ and $k_{\text {cat }}$ values, the average $\mathrm{V}_{\max }$ was represented against substrate concentration and fitted to a single rectangular hyperbola function using SigmaPlot 10.0, where parameter $a$ was equal to $\mathrm{k}_{\text {cat }}$ and parameter $b$ was equal to $K_{\mathrm{m}}$.

HPLC analysis. The reactions were analyzed by reverse phase chromatography (HPLC) on an equipment composed by a tertiary pump (Varian-Agilent Technologies, USA) coupled to an autosampler (Merck Millipore, MA, USA) and using an ACE C18 PFP (pentafluorophenyl, 15 $\mathrm{cm} \times 4.6 \mathrm{~mm}$ ) column at $45^{\circ} \mathrm{C}$. Detection was performed with a photodiode array detector (PDA) (Varian, Agilent Technologies, USA). The mobile phase was $70 \%$ methanol and $30 \% d d \mathrm{H}_{2} \mathrm{O}$ (both with $0.1 \%$ acetic acid) at a flow rate of $0.8 \mathrm{~mL} / \mathrm{min}$. The reaction was quantified at $268 \mathrm{~nm}$ (based on HPLC standards). For the $15 \mathrm{~min}$ reaction, the reaction mixture contained $6.6 \mathrm{nM}$ of purified enzyme, $1 \mathrm{mM}$ naphthalene, $20 \%$ acetonitrile and $1 \mathrm{mM} \mathrm{H}_{2} \mathrm{O}_{2}$ in $100 \mathrm{mM}$ potassium phosphate $\mathrm{pH} 7.0$ (final volume of $1 \mathrm{~mL}$ ). The reaction was started by the addition of the $\mathrm{H}_{2} \mathrm{O}_{2}$ and stopped by adding $20 \mu \mathrm{L}$ of $37 \% \mathrm{HCl}$; a $10 \mu \mathrm{L}$ sample was injected and analyzed. For the longer reactions, the conditions were as described above, but the reaction was not stopped with $\mathrm{HCl}$. A $10 \mu \mathrm{L}$ sample was injected and analyzed at different times ( 1 to $270 \mathrm{~min}$ ). To determine the kinetic values for 1-naphthol, the reaction was performed using $40 \mathrm{nM}$ of the pure enzymes, $1 \mathrm{mM}$ naphthol, $20 \%$ acetonitrile and 1 
$\mathrm{mM} \mathrm{H} \mathrm{H}_{2}$ in $100 \mathrm{mM}$ potassium phosphate $\mathrm{pH} 7.0$ (final volume of 0.2 $\mathrm{mL})$. Standard deviations were lower than $5 \%$ in all experiments.

MALDI-TOF-MS analysis and $\mathrm{pl}$ determination. Experiments were performed on an Autoflex III MALDITOF-TOF instrument with a smartbeam laser (Bruker Daltonics). A laser power just above the ionization threshold was used in order to acquire the spectra and the samples were evaluated in the positive-ion detection mode. External calibration was performed, using the BSA from Bruker, covering the range $15,000-70,000 \mathrm{Da}$. To determine UPO's $\mathrm{pl}, 8 \mu \mathrm{g}$ of purified enzyme were subjected to two-dimensional electrophoresis gel. These determinations were carried out at the Proteomic and Genomic Services at the CIB (CSIC, Spain).

LC/MS. A mass spectrometer with a hybrid Q-TOF mass analyzer (QSTAR, ABSciex, MA, USA) was used. The ionization source was electrospray $(\mathrm{ESI})$ with methanol as the ionization phase and the inlet system was direct injection in a HPLC 1100 (Agilent Technologies, USA). The resolution of the assay was 9,000 FWHM (full width at half maximum), the accuracy was $5-10 \mathrm{ppm}$ and it was carried out in negative mode.

DNA sequencing. Plasmid-containing variant upo1 genes were sequenced on an ABI 3730 DNA Analyzer/Applied Biosystems Automatic Sequencer by Secugen (Spain). The primers used were: RMLN; apo1secdir (gaaggcgacgccagtatgacc); apo1secrev (ggtcatactggcgtcgccttc) and RMLC.

\section{Computational analysis:}

System preparation for molecular modeling. The structure of wild-type UPO1 (purified from $A$. aegerita culture) at a resolution of $2.1 \AA$ (Protein Data Bank Europe [PDB] accession number 2YOR) was used as departure point for $\mathrm{PaDa}-\mathrm{I}$ and JaWa variants modeling. ${ }^{[27]}$ The wild-type UPO1 crystal was used. Five different mutations (V57A-L67F-V75II248V-F311L) were introduced to model PaDa-I variant, with two additional mutations (G241D-R257K) for JaWa. As the optimal pH for DMP and naphthalene activity is $\sim 7$, mutated structures (and the UPO1 crystal) were prepared accordingly using the Schrodinger's Protein Preparation Wizard and the $\mathrm{H}_{++}$web server. ${ }^{[37,38]}$ All acidic residues were deprotonated except Asp85. Histidines were $\delta$-protonated, with the exception of His82 ( $\varepsilon$-protonated) and His118 and His251 (doubleprotonated). To relax the systems after mutation, and to investigate its possible effect on the protein structure, UPO1 variants were subjected to 5 ns molecular dynamics (MD) with Desmond. ${ }^{[39]}$ Finally, the heme site was modeled as compound I after being fully optimized in the protein environment with quantum mechanics/molecular mechanics (QM/MM) using QSite. ${ }^{[40]}$ DMP and naphthalene molecules were also optimized with Jaguar ${ }^{[41]}$ at the DFT/M06 level with the $6-31 \mathrm{G}^{\star *}$ basis and PBF implicit solvent in order to obtain their electrostatic potential atomic charges.

Protein Energy Landscape Exploration (PELE) computational analysis. Once PaDa-I and JaWa structures were prepared and ligands optimized, heme binding site and global protein surface exploration were performed with PELE, a Monte Carlo algorithm capable of effectively sampling the protein-ligand conformational space. ${ }^{[37]}$ For the protein surface exploration, ligands were placed manually in 20 initial random positions on the protein's surface and the ligand allowed to explore freely. For each system, 160 independent 48-h simulations were performed. On the other hand, for the heme binding site exploration, the substrates were placed manually in identical positions at the entrance of the heme-access channel. From there, ligands were spawned inside the protein by PELE. Once the ligand's center of mass reached $<5 \AA$ to the heme catalytic oxygen, it was free to explore the active site cavity with 96 independent 48-h simulations.

QM/MM simulations. Hybrid quantum mechanics/molecular mechanics (QM/MM) calculations were carried out for two different purposes: to identify the highest oxidizable surface residue in UPO1 protein, and to investigate the mutations effect on naphthalene oxidation. For the first purpose, a QM/MM calculation was performed in wild-type UPO1 by including all potential surface oxidation sites $(9$ tyrosines and 1 tryptophan) in the quantum region, subtracting one electron and computing the spin density. A subsequently QM/MM pairwise comparison was performed with residues that showed a clear preference to be oxidized (Trp24 and Tyr47). Calculations were performed at the DFT M06-L(lacvp*)/OPLS level. On the other hand, to study the differences between PaDa-I and JaWa naphthalene oxygenation, five ionization energies of the substrate (located on the binding site of the variants) were computed. Energies were taken from single-point calculations using the B97-D3(cc-pVTZ(-f)++) basis set. All QM/MM calculations were performed using Qsite.

\section{Acknowledgements}

We thank Paloma Santos Moriano (ICP, CSIC, Spain) for assistance with the HPLC and LC/MS analysis, and Jesper Vind (Novozymes, Denmark) and Angel T. Martinez (CIB, CSIC, Spain) for helpful discussions. This work was supported by the European Commission projects Indox-FP7-KBBE-2013-7613549 and Cost-Action CM1303-Systems Biocatalysis, and the National Projects Dewry [BIO201343407-R], Cambios [RTC2014-1777-3] and OXYdesign [CTQ2013-48287-R].

Keywords: Directed evolution $\cdot 1$-naphthol $\bullet$ unspecific peroxygenase $\cdot$ peroxygenase activity $\bullet$ peroxidative activity

[1] G. Booth, in Ullmann's Encyclopedia of Industrial Chemistry, Vol. 23, Wiley-VCH Verlag GmbH \& Co. KGaA, 2000, pp. 671-723.

[2] K. R. Jegannathan, P. H. Nielsen, J. Clean. Prod. 2013, 42, 228240.

[3] K. Kudo, T. Ohmae, A. Uno, United States Patent 1976, 3,935,282.

[4] L. Schuster, B. Seid, United States Patent 1979, 4,171,459.

[5] I. Calinescu, R. Avram, Revista de chemie 1994, 45, 97-103.

[6] I. Calinescu, R. Avram, H. lovu, Revista de chemie 1994, 45, 299305.

[7] I. Calinescu, R. Avram, Revista de chemie 1994, 45, 865-867.

[8] J. B. van Beilen, W. A. Duetz, A. Schmid, B. Witholt, Trends Biotechnol. 2003, 21, 170-177.

[9] B. Buhler, A. Schmid. J. Biotechnol. 2004, 113, 183-210

[10] V. B. Urlacher, R. D. Schmid, Curr. Opin. Chem. Biol. 2006, 10, 156-161.

[11] R. Ullrich, M. Hofrichter, Cell. Mol. Life. Sci. 2007, 64, 271-293.

[12] H. Joo, Z. Lin, F. H. Arnold, Nature 1999, 399, 670-673.

[13] P. C. Cirino, F. H. Arnold, Angew. Chem. Int. Ed. 2003, 42, 32993301.

[14] P. Meinhold, M. W. Peters, A. Hartwick, A. R. Hernandez, F. H. Arnold, Adv. Synth. Catal. 2006, 348, 763-772.

[15] P. S. Coelho, E. M. Brustad, A. Kannan, F. H. Arnold, Science 2013, 339, 307-310

[16] K. A. Canada, S. Iwashita, H. Shim, T. K. Wood, J. Bacteriol. 2002, 184, 344-349.

[17] L. Rui, Y. M. Kwon, A. Fishman, K. F. Reardon, T. K. Wood, Appl. Environ. Microb. 2004, 70, 3246-3252. 
[18] J. Garikipati, A. M. Mclver, T. L. Peeples, Appl. Environ. Microb. 2009, 75, 6545-6552.

[19] R. Ullrich, J. Nüske, K. Scheibner, J. Spantzel, M. Hofrichter, Appl. Environ. Microb. 2004, 70, 4575-4581.

[20] M. Hofrichter, R. Ullrich, M.J. Pecyna, C. Liers, T. Lundell T. Appl. Microbiol. Biot. 2010, 87, 871-897.

[21] M. Hofrichter, R. Ullrich, Curr. Opin. Chem. Biol. 2014, 19, 116-125.

[22] M. Kluge, R. Ullrich, C. Dolge, K. Scheibner, M. Hofrichter, Appl. Microbiol. Biotechnol. 2009, 81, 1071-1076.

[23] P. Molina-Espeja, E. Garcia-Ruiz, D. Gonzalez-Perez, R. Ullrich, M. Hofrichter, M. Alcalde, Appl. Environ. Microb. 2014, 80, 3496 3507

[24] P. Molina-Espeja, S. Ma, D.M. Mate, R. Ludwig, M. Alcalde, Enz. Microb. Tech. 2015, 73-74, 29-33.

[25] H. Zollinger, in Diazo Chemistry I, Wiley-VCH Verlag GmbH \& Co. KGaA, 2004, pp.1-13.

[26] E. Aranda, R. Ullrich, M. Hofrichter, Biodegradation 2010, 21, $267-$ 281.

[27] K. Piontek, E. Strittmatter, R. Ullrich, G. Gröbe, M.J. Pecyna, M. Kluge, K. Scheibner, M. Hofrichter, D. A. Plattner, J. Biol. Chem.2013, 288, 34767-34776.

[28] A. Madadkar-Sobhani, V. Guallar. Nucleic Acids Res. 2013, 41, W322-W328.

[29] D. Linde, R. Pogni, M. Cañellas, F. Lucas, V. Guallar, M.C Baratto, A. Sinicropi, V. Sáez-Jiménez, C. Coscolín, A. Romero, F.J. Medrano, F.J. Ruiz-Dueñas, A.T. Martínez. Catalytic surface radical in dye-decolorizing peroxidase: a computational, spectroscopic and directed mutagenesis study. Biochem. J. 2015 466, 253-262.

[30] D. Kumar, B. Karamzadeh, G.N. Sastry, S.P. de Visser. J. Am. Chem Soc. 2010, 132, 7656-7667.

[31] A.J.M. Ribeiro, D. Santos-Martins, N. Russo, M.J. Ramos, P.A. Fernandes, ACS Catal. 2015, 5, 5617-5626.

[32] B. Valderrama, M. Ayala, R. Vazquez-Duhalt, Chem. Biol. 2002, 9 , 555-565.

[33] M. Hofrichter, H. Kellner, M.J. Pecyna, R. Ullrich. Adv Exp Med Biol. 2015, 851, 341-68.

[34] H. Zhao, L. Giver, Z. Shao, J. A. Affholter, F. H. Arnold, Nat Biotech. 1998, 16, 258-261.

[35] M. Alcalde, Methods Mol. Biol. 2010, 634, 3-14.

[36] M. G. Kluge, R. Ullrich, K. Scheibner, M. Hofrichter, Appl. Microbiol. Biotechnol. 2007, 75, 1473-1478.

[37] G.M. Sastry, M. Adzhigirey T. Day, R. Annabhimoju, W. Sherman. J. Comput. Aided Mol. Des. 2013, 27, 221-234.

[38] R. Anandakrishnan, B. Aguilar, A.V. Onufriev. Nucleic Acids Res. 2012, 40, W537-W541.

[39] Desmond Molecular Dynamics System, version 2.2, D. E. Shaw Research, New York, NY, 2009. Maestro-Desmond Interoperability Tools, version 2.2, Schrödinger, New York, NY.

[40] QSite, version 5.7, Schrödinger, LLC, New York, NY, 2011.

[41] Jaguar, version 8.1, Schrödinger, LLC, New York, NY, 2013. 
Entry for the Table of Contents (Please choose one layout)

Layout 1:

\section{FULL PAPER}

An aromatic peroxygenase was engineered to synthesis 1 naphthol. By modifying the ratio of peroxidative and peroxygenase activity, the evolved enzyme had total turnover numbers of 50,000, with high selectivity. This biocatalyst represents a promising one-pot alternative to replace the standard aggressive and contaminant chemical processes that currently prevail, while complying with the industrial demands for 1-naphthol synthesis.
Patricia Molina-Espeja, Marina Cañellas, Francisco J. Plou, Martin Hofrichter, Fatima Lucas, Victor Guallar, and Miguel Alcalde

Page No. - Page No.

Synthesis of 1-Naphthol by a Natural Peroxygenase Engineered by Directed Evolution

Layout 2:

\section{FULL PAPER}

((Insert TOC Graphic here; max. width: $11.5 \mathrm{~cm}$; max. height: $2.5 \mathrm{~cm}$ ))

Author(s), Corresponding Author(s)*

Page No. - Page No.

Title

Text for Table of Contents 